\title{
Hybrid endo-laparoscopic management of sigmoid colon perforation due to an accidentally ingested denture
}

\author{
S. H. R. Sanjeewa ${ }^{1}$, V. Sanjeev ${ }^{1}$, G. M. D. Kariyawasam ${ }^{1}$,M. A. C. Lakmal ${ }^{1}$,G. P. U. P. de Silva ${ }^{1}$, \\ B. D Gamage ${ }^{2}$ \\ ${ }^{1}$ University Surgical Unit, Colombo South Teaching Hospital
}

Keywords: Hybrid; endoscopy; laparoscopy; ingested denture; bowel perforation

\section{Introduction}

Denture ingestion is one of the commonest ingested foreign bodies we encounter in surgical casualty. The concern is that most dentures are radiolucent and difficult to identify on conventional imaging which may delay diagnosis. Here we present a case of a 46-year-old female who was admitted with clinical and radiological signs of bowel perforation. X-ray supine abdomen and the non-contrast CT imaging failed to provide a conclusive diagnosis. Diagnostic laparoscopy revealed a mid-sigmoid perforation with partial protrusion of the denture. Colonoscopic retrieval of the denture and intracorporeal suturing of the sigmoid perforation were performed. The patient had an uneventful recovery. We suggest minimal acess interventions are both diagnostic and therapeutic in the management of complications of ingested foreign bodies.

\section{Case presentation}

A 46-year-old female presented with lower abdominal pain for 4 hours duration. She recalled swallowing her loose denture while taking pills 3 days before the admission. She was tachycardic. Abdominal examination revealed tenderness over the lower abdomen. Erect chest X-ray showed air under the diaphragm. Though the NCCT abdomen confirmed pneumoperitoneum it couldn't conclusively identify the foreign body.

The decision was made to proceed with diagnostic laparoscopy. The pointed end of the denture was seen through a perforation of the mid sigmoid colon [Figure 1]. A moderate amount of purulent fluid was present in the peritoneal cavity. No gross faecal contamination was seen. The denture was carefully pushed back into the sigmoid colon and the decision was taken to attempt endoscopic retrieval of the denture and laparoscopic repair of the perforation. The sigmoid colon was clamped just distal to the perforation with a non-

Correspondence: Chamila Lakmal

E-mail: lakmalmac@gmail.com

(i) https://orcid.org/0000 000338677861

Received: 08-01-2021 Accepted: 30-03-2021

DOI: http://doi.org/10.4038/sljs.v39i1.8793 traumatic intestinal grasper to avoid unnecessary bowel distension during colonoscopy. The denture was grasped with snare forceps [Boston Scientific Microvasive ${ }^{\circledR}$, rotatable snare forceps] and withdrawn carefully under direct vision without damaging the colonic wall Figure 2]. Perforation was repaired intracorporeally with $3 / 0$ polydioxanone [PDS] interrupted sutures. A thorough washout of the peritoneal cavity was done with normal saline.

The patient had an uneventful recovery and discharged on the third postoperative day.

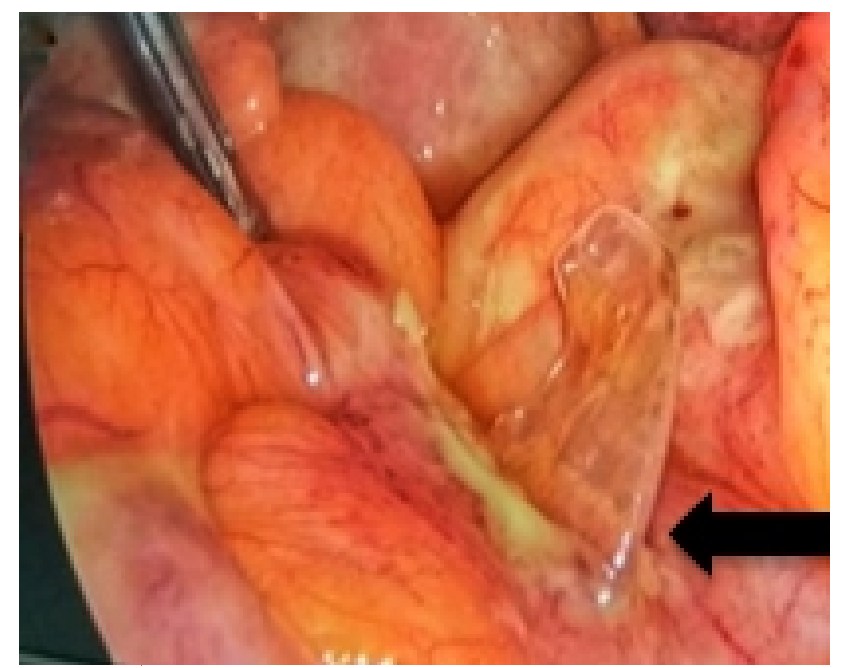

Figure 1. Part of the denture is seen through the perforation of the sigmoid colon

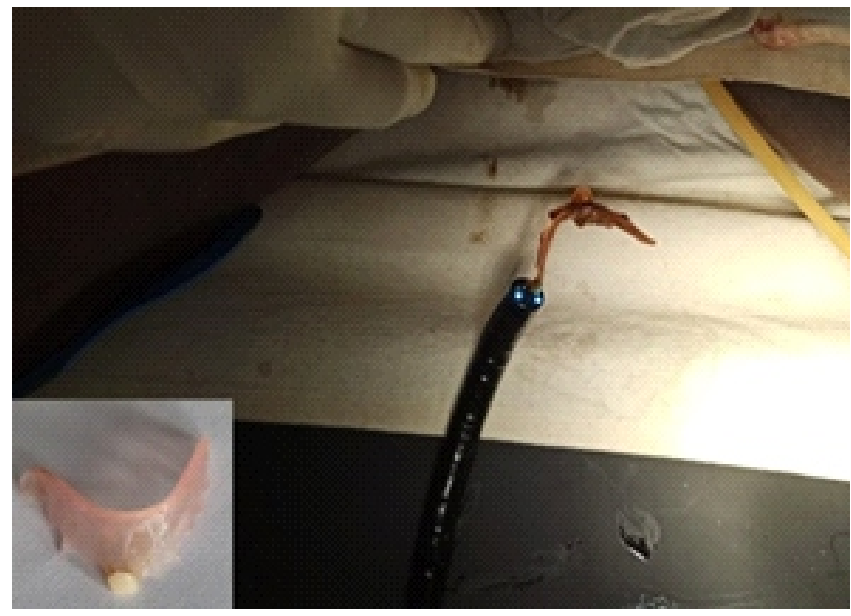

Figure 2. Endoscopic retrieval of the denture 


\section{Discussion}

Ingestion of foreign bodies is a common presentation to surgical casualty. Dentures are among the commonest such ingested items [1]. Studies estimated that the majority [80$90 \%$ ] of these will pass through the digestive tract without complications. However, necrosis, perforation, migration to close-by organs, haemorrhage and bowel obstruction have been reported. Gastrointestinal perforation is a rare complication, occurring in less than $1 \%$ of the cases of foreign body ingestion [2]. Perforation frequently occurs at sites of acute angulation and narrowing. Ileo-caecal junction and sigmoid colon are the commonest sites where perforation occurs.

Computed Topography [CT] is considered the best method to accurately locate the site of foreign bodies [2]. In the event of perforation, CT has shown the perforation and the cause of perforation with $86 \%$ accuracy [2] Most of the dentures are made from polymethylmethacrylate [PMMA] which is radiolucent. Therefore it is difficult to visualize on conventional imaging [3]. In our case non-contrast, CT imaging failed to localize the denture conclusively.

Management options following ingestion of dentures are close observation, endoscopic extraction and surgery. When there are no complications at presentation, the denture is small in size, its shape does not risk causing injury to the bowel wall [e.g. not pointed; no sharp edges] and when the item is distal to the ligament of Trietz at presentation, observation can be considered [4].

Approximately 1-14\% of ingested foreign bodies will necessitate surgical intervention. Indications for surgery are failed endoscopic retrieval and the presence of complications [4]. Our patient required surgery as she presented with evidence of visceral perforation.

Complicated foreign bodies particularly with bowel perforation require surgery. Most perforations need open surgery. Combined laparoscopy and trans-anal approach for retrieval of a foreign body in the distal colon has been reported [5]. The foreign body has been pushed down from the distal colon into the rectum and retrieved per anally using an anoscope.

Not many cases of hybrid endo-laparoscopic management of foreign bodies with visceral perforation have been reported. It avoids a major laparotomy, gives early recovery with less wound-related complications and good cosmesis as well. As we demonstrated, this method is technically feasible and safe.

All authors disclose no conflict of interest. The study was conducted in accordance with the ethical standards of the relevant institutional or national ethics committee and the Helsinki Declaration of 1975, as revised in 2000 .

\section{References}

1. Sarmast AH, Showkat HI, Patloo AM et al. Gastrointestinal Tract Perforations Due to Ingested Foreign Bodies; A review of 21 cases. Brit Jour of Medi Practi. 2012 Sep 1;5[3].

2. Kim SH, Shin SS, Jeong YY et al. Gastrointestinal tract perforation: MDCT findings according to the perforation sites. Kore Jour of Radio. 2009 Feb 1;10[1]:63-70. https://doi.org/10.3348/kjr.2009.10.1.63.

3. Borofsky S, Taffel M, Khati $\mathrm{N}$ et al. The emergency room diagnosis of gastrointestinal tract perforation: the role of CT. Emer Radio. 2015 Jun 1;22[3]:315-27.

https://doi.org/10.1007/s10140-014-1283-4

4. Chia DK, Wijaya R, Wong A et al. Laparoscopic management of complicated foreign body ingestion: a case series. Inter sur. 2015 May;100[5]:849-53. https://doi.org/10.9738/INTSURG-D-14-00238.1

5. Rispoli G, Esposito C, Monachese D et al. Removal of a foreign body from the distal colon using a combined laparoscopic and endoanal approach. Dise of the colo \& rect. 2000 Nov 1;43[11]:1632-4.

https://doi.org/10.1007/BF02236754

\section{Learning Points:}

- Approximately 1-14\% of ingested foreign bodies will necessitate surgical intervention.

- Laparoscopy has a diagnostic and therapeutic value in management of ingested foreign bodies with complications.

- Hybrid endo-laparoscopic management of foreign bodies with visceral perforation avoids a major laparotomy, gives early recovery with less wound-related complications and good cosmesis, and is technically feasible and safe. 